\title{
Biomembrane elastic response to intercalation of amphiphiles
}

\author{
E. Farge, M. Bitbol, and P. F. Devaux \\ Institut de Biologie Physico-Chimique, 13 rue Pierre et Marie Curie, F-75005 Paris, France
}

Received March 19, 1990/Accepted in revised form August 6, 1990

\begin{abstract}
A model of the elastic behavior of a biomembrane in response to intercalation of amphiphiles into the bilayer is developed. This model takes into account the bilayer couple hypothesis (Sheetz and Singer 1974), and assumes that incorporation of amphiphiles into one layer of the membane exerts mechanical work on the elastic biomembrane. The model accounts for an apparent experimental discordance noted by several authors: the variation in area observed upon incorporating amphiphiles is smaller by a factor of about 2 than the variation expected using previous models.
\end{abstract}

Key words: Elastic membrane - Bilayer couple hypothesis - Shape change - Transverse asymmetry - Erythrocyte membrane

\section{Introduction}

In good qualitative agreement with the bilayer couple hypothesis (Sheetz and Singer 1974) and geometrical analysis of red blood cell shape transformations (Beck 1978), intercalation of drugs or exogenous phospholipids in the outer leaflet of the erythrocyte bilayer results in echinocytosis (Lange and Slayton 1982; Kuypers et al. 1984; Ferrell et al. 1985; Allan et al. 1989). Conversely, depletion of the outer layer, or intercalation of amphiphiles in the inner layer, produces stomatocytosis (Isomaa et al. 1987; Rosso et al. 1988; Haest et al. 1981). However, quantitative assessments have shown that the expansion (or the constriction) of the treated layer is not as large as one would expect taking into account the number of molecules incorporated (or removed), and the average area per molecule (Ferrell et al. 1985; Haest et al. 1981; Allan et al. 1989). The authors observed variations in surface area which were half the expected values. Several explanations have been put forward in the above quoted papers to explain this differ-

Offprint requests to: E. Farge ence. Some of these explanations speculated that cholesterol redistributed across the bilayer. Other explanations concern the mechanical properties of the membrane. In the present paper, we propose a simple mechanical analysis of the membrane elastic response to intercalation (or depletion) of amphiphiles into the bilayer, which accounts for the numerical discrepancy between observed and predicted variations of area.

\section{Theory}

We consider that the membrane behaves as if it were made of two coupled layers. For two coupled layers, bending (or shape change) is associated with the compression or dilation of each leaflet. So each layer is described as a two-dimensional elastic surface, with its own set of elastic moduli: we shall only make use of the elastic area compressibility moduli $K_{i}\left(K_{1}\right.$ for the inner layer, and $K_{2}$ for the outer layer). For the sake of simplicity, we shall focus our attention on the situation wherein amphiphiles (drugs, phospholipids or lysophospholipids) are intercalated in the outer layer. The results obtained in this case will easily be transposed to the other situation where amphiphiles are depleted from the outer layer.

The mechanical work needed to vary the area of each elastic surface can be written as follows (Evans 1974):

$$
W_{1}=1 / 2 K_{1} \alpha_{1}^{2} ; \quad W_{2}=1 / 2 K_{2} \alpha_{2}^{2}
$$

where

$\alpha_{1}=\frac{S_{1}-S_{01}}{S_{01}} \quad$ and $\quad \alpha_{2}=\frac{S_{2}-S_{02}}{S_{02}}$.

$S_{01}$ and $S_{02}$ are respectively the areas of the inner and the outer leaflet in their unconstrained state, whereas $S_{1}$ and $S_{2}$ are the actual areas of same leaflets in any given state.

Intercalation or depletion of amphiphiles in the outer layer of the bilayer provides an external mechanical work $\mathrm{d} W^{\text {ext }}$, producing the bending of the membrane and its 
shape change (echinocytosis in the case we are interested in). This work is due to the change of the unconstrained outer area $S_{02}$ and can be written:

$\mathrm{d} W^{\text {ext }}=\left.\frac{\partial W_{2}}{\partial S_{02}}\right|_{\alpha_{2}} \mathrm{~d} S_{02}$.

The mechanical work $\mathrm{d} W^{\text {rec }}$ received by the two leaflets expresses the resistance of the membrane to bending. It is due to compression (or extension) of their surface relative to the unconstrained area, and is the following:

$\mathrm{d} W^{\mathrm{rec}}=\left.\frac{\partial W_{1}}{\partial \alpha_{1}}\right|_{S_{01}} \mathrm{~d} \alpha_{1}+\left.\frac{\partial W_{2}}{\partial \alpha_{2}}\right|_{S_{02}} \mathrm{~d} \alpha_{2}$.

The bending of the bilayer as a consequence of the unconstrained area $S_{02}$ variation is a purely mechanical and adiabatic transformation. Therefore, the energy conservation leads to a conservation equation for the mechanical work wherein external given work must be equal to received work:

$\left.\frac{\partial W_{1}}{\partial \alpha_{1}}\right|_{S_{01}} \mathrm{~d} \alpha_{1}+\left.\frac{\partial W_{2}}{\partial \alpha_{2}}\right|_{S_{02}} \mathrm{~d} \alpha_{2}=\left.\frac{\partial W_{2}}{\partial S_{02}}\right|_{\alpha_{2}} \mathrm{~d} S_{02}$

or, taking (1) into account:

$K_{1} \alpha_{1} \mathrm{~d} \alpha_{1}+K_{2} \alpha_{2} \mathrm{~d} \alpha_{2}=-K_{2} \alpha_{2}\left(\alpha_{2}+1\right) \frac{\mathrm{d} S_{02}}{S_{02}}$.

One may go one step further, by noticing that, since the two layers are coupled, extension of $S_{02}$ leads the membrane to a bending equilibrium state. At this bending equilibrium, the stresses of each layer must be equal:

$K_{1} \alpha_{1}=K_{2} \alpha_{2} \quad$ or $\quad K_{1} \mathrm{~d} \alpha_{1}=K_{2} \mathrm{~d} \alpha_{2}$.

Then, (5) becomes:

$\frac{\mathrm{d} \alpha_{2}}{\alpha_{2}+1}=-\frac{K_{1}}{K_{1}+K_{2}} \frac{\mathrm{d} S_{02}}{S_{02}}$.

Finally, taking (2) into account, (6) can be written:

$\frac{\mathrm{d} S_{2}}{S_{2}}=\frac{K_{2}}{K_{2}+K_{1}} \frac{\mathrm{d} S_{02}}{S_{02}}$.

Relative variations of the area are on the order of a few percents: this justifies the infinitesimal notation employed in (7).

Formula (7) shows that the relative variation of the outer area $\mathrm{d} S_{2} / S_{2}$ is not equal to the relative variation of the corresponding unconstrained area $\mathrm{d} S_{02} / S_{02}$. In the situation we are interested in, the variation of the unconstrained area actually equals the average area per molecule times the number of incorporated, or extracted, molecules. Thus, we find that, taking into account the elastic properties of both layers of the membrane, the variation of the outer layer area is necessarily smaller than the average area per (added or extracted) molecule times the number of molecules.

Moreover, if $K_{1}=K_{2}$, we have $\mathrm{d} S_{2} / S_{2}=1 / 2 \mathrm{~d} S_{02} / S_{02}$, which is in good agreement with the measurements performed by Ferrell et al. (1985) and by Allan et al. (1989).

\section{Discussion}

Several explanations have been put forward in order to account for the difference between the experimental evaluations of the outer leaflet expansion, and the theoretical estimates based on the cross-sectional area of the intercalated molecules. We discuss hereafter two major proposals:

\section{The cholesterol hypothesis}

It has been suggested (Haest et al. 1981) that cholesterol translocation could compensate for the imbalance induced by intercalation (or depletion) of amphiphiles in the outer leaflet. This model is based on the assumption that the transbilayer movement of cholesterol is very fast (see Lange et al. 1981). But these data have recently been challenged (Van Meer 1987). Moreover, a direct study of the effect of cholesterol on lysolecithin-induced red cell crenation (Lange and Slayton 1982) showed that the amount of lysolecithin required for echinocytosis decreases as the membrane cholesterol content increases. One would expect the opposite if cholesterol redistribution accross the bilayer would occur, and act as a partial compensatory mechanism for the lysolecithin-induced excess of the outer leaflet area: the less cholesterol present in the membrane, the less efficient should be the compensation.

\section{The dilation hypothesis}

The other main explanation is purely mechanical, involving an expansion of the inner lipid leaflet in response to the outer leaflet expansion (Allan et al. 1989). But here, one must realize that the outer leaflet expansion following intercalation of amphiphiles is related to an increase of the unconstrained area $S_{02}$, whereas the actual area $S_{2}$ is likely to increase less than $S_{02}$. As for the hypothetical inner leaflet expansion, it can be related to an increase of the actual area $S_{1}$ with respect to a fixed $S_{01}$. In this case, one would have $\alpha_{2}<0$ and $\alpha_{1}>0$, and thus, the tensions in the two leaflets would have opposite directions. Hence, a torque would persist, and the bilayer would not be in mechanical equilibrium. Bending would result, and lead the membrane to an equilibrium state with both layers compressed.

Intuitively, if the surface does not extend - or diminish - as much as it should, elastic compression - or dilation - may effectively occur, and one must take into accout the elastic properties of the biomembrane. We have developed a model of elastic response of a biomembrane to amphiphiles intercalation, which resolves quantitatively experimental discordances in the above cited publications. The present model allows us to make some considerations on an hypothetic mechanical asymmetry in biological membrane, on its consequences and its origin. 


\section{The bilayer elastic response}

In our model, the bilayer couple hypothesis is expressed by the equilibrium condition $K_{1} \alpha_{1}=K_{2} \alpha_{2}$. This condition expresses equality between the tensions $T_{1}$ and $T_{2}$ of the two layers, which is the definition of mechanical equilibrium between two coupled layers related by shape changes: this is the bending equilibrium state. Then we write the external mechanical work received by the bilayer because of amphiphiles incorporation into a leaflet. Writing the energy conservation equation as an equality between received and external work, and introducing the bilayer couple hypothesis leads us to show that when $S_{02}$ is increased by intercalation of amphiphiles, then both $\alpha_{1}$ and $\alpha_{2}$ are negative, namely both leaflets are compressed with respect to their unconstrained area. Depletion of the outer leaflet and decrease of $S_{02}$ would rather lead to $\alpha_{1}$ and $\alpha_{2}$ positive and to an expansion with respect to the unconstrained area.

This model allows us to give a quantitative answer to the publications mentioning a discrepancy of about 2 between $\mathrm{d} S_{02} / S_{02}$ (the expected value of area variation) and $\mathrm{d} S_{2} / S_{2}$ (the observed value). Assuming $K_{1}=K_{2}$, and introducing this condition in (7), one finds: $\mathrm{d} S_{2} / S_{2}=(1 / 2)$ $\mathrm{d} S_{02} / S_{02}$, which is the result obtained in the experiments, account being taken of the inaccuracies.

\section{A putative mechanical asymmetry}

Moreover, if one considers the fact that in the quoted experiments the factor is not exactly equal to 2 , our model provides the possibility of estimating a putative mechanical asymmetry of compressibility moduli across the membrane. The asymmetric transverse repartition of the phospholipids is already known to involve physical asymmetric properties across the bilayer: this is the case for the lateral diffusion of phospholipids in the membrane which is faster by about a factor 5 in the inner layer than in the outer layer of the red blood cell membrane (For a review see Zachowski and Devaux 1989). Such an asymmetry could be the cause of differences in the elastic physical properties between the two layers. It has been shown in model membranes that there is a close correlation between phospholipid composition and elastic compression moduli (Evans and Needham 1987). Each layer, characterized by its own phospholipid composition, could consequently have its own elastic behavior, and thus different elastic moduli $K_{1}$ and $K_{2}$.

Deuling and Helfrich have pointed out that such an asymmetry may play a role in the spontaneous curvature of the membrane (Deuling and Helfrich 1976). Also it has been shown theoretically, with a bending energy minimisation method (Svetina and Zeks 1989), that the value of the difference between external area of the two layers can be correlated to some red blood cell shape changes, like discocyte or cup formation. A similar hypothesis has been put forward to explain vesicle shape changes (Sackmann et al. 1986).

Thus, one could imagine that mechanical asymmetry could control changes in membrane curvatures, and pos- sibly play a role in phenomena involving local shape changes which are involved in the early stage of endocytosis. In conclusion, modulation of the difference in area between the inner and outer layers of eukaryotic cell membranes via the putative aminophospholipid translocase may play an important physiological role (Devaux 1990).

The mechanical asymmetry could either be purely related to the properties of the lipid bilayer or involve the cytoskeleton. As an instance of the second case, one can consider an experiment (Haest et al. 1980) wherein the addition of diamide cross-links the spectrin network and decreases its compliance, increasing the $K_{1}$ value. In this situation, the effective area variation under amphiphile depletion of the outer layer $\left(\mathrm{d} S_{2} / S_{2}\right)$ tends to 0 . Using formula (7) with $K_{1} \gg K_{2}$ leads to the same result. Our model, taking into account the elastic asymmetry is, thus, in good agreement with this diamide experiment too, where the cytoskeleton is involved as the internal layer. Noticing that echinocytic shape change can be conceptualised as an expansion of the bilayer in respect to the cytoskeleton, properties of the cytoskeleton may also be involved in mechanical asymmetry for such a transformation.

A consequence of our model is that shape changes induced by incorporation or depletion of amphiphiles respectively lead the membrane to a compressed or dilated equilibrium state. Consequently, the surface tension of the membrane should be non-zero. A non-zero tension is known to inhibit flickering phenomenon, the thermal fluctuations of the membrane (Brochard and Lennon 1975). According to our model, shape change should then imply inhibition of flickering. However, because of the very low value of the bending elastic modulus - about $10^{-20} \mathrm{~N} \cdot \mathrm{m}-$ tensions due to bending are probably to small to involve any detectable inhibition of flickering. This could be the reason why the discocyte-echinocyte shape transformation does not experimentally exhibit any inhibition of the flickering (Zeman et al. 1990).

Acknowledgements. This work was supported by grants from the Centre National de la Recherche Scientifique (UA 526) and the Universite Paris VII. E.F. is a recipient of a followship from the Ministère de la Recherche et de la Technologie.

\section{References}

Allan D, Hagelberg C, Kallen KJ, Haest CWM (1989) Echinocytosis and microvesiculation of human erythrocytes induced by insertion of merocyanine 540 into the outer membrane leaflet. Biochim Biophys Acta 986:115-122

Beck JS (1978) Relations between membrane monolayers in some red blood cell shape transformations. J Theor Biol 75:487-501

Brochard F, Lennon JF (1975) Frequency spectrum of the flicker phenomenon in erythrocytes. J Phys 36:1035-1047

Deuling HJ, Helfrich H (1976) The curvature energy of fluid membranes: a catalogue of vesicule shapes. J Phys 37:1335-1345

Devaux PF (1990) The aminophospholipid translocase: a transmembrane lipid pump; physiological significance. News Physiol Sci 5:53-58 
Evans EA (1974) Bending resistance and chemically induced moments in membrane bilayers. Biophys $\mathrm{J}$ 14:923-931

Evans EA, Needham D (1987) Physical properties of surfactant bilayer membranes: thermal transitions, elasticity, rigidity, cohesion and colloidal interactions. J Phys Chem 91:4219-4228

Ferrell JE, Kong-Joo Lee Jr, Huestis WH (1985) Membrane bilayer balance and erythrocyte shape: a quantitative assessment. Biochemistry $24: 2849-2857$

Haest CWM, Fischer TM, Plasa G, Deuticke B (1980) Stabilisation of erythrocyte shape by a chemical increase in membrane shear stiffness. Blood Cells 6:539-553

Haest CWM, Plasa G, Deuticke B (1981) Selective removal of lipids from the outer membrane layer of human erythrocytes without hemolysis. Consequences for bilayer stability and cell shape. Biochim Biophys Acta 649:701-708

Isomaa B, Hägerstrand H, Paatero G (1987) Shape transformations induced by amphiphiles in erythrocytes. Biochim Biophys Acta 899:93-103

Kuypers FA, Roelofsen B, Berendsen W, Op Den Kamp JAF, Van Deenen LLM (1984) Shape changes in human erythrocytes induced by replacement of the native phosphatidylcholine with species containing various fatty acids. J Cell Biol 99:2260-2267

Lange Y, Slayton JM (1982) Interaction of cholesterol and lysophosphatidylcholine in determining red cell shape. J Lipid Res 23: $1121-1127$
Lange Y, Dolde J, Steck TL (1981) The rate of transmembrane movement of cholesterol in the human erythrocyte. J Biol Chem $256: 5321-5323$

Rosso J, Zachowski A, Devaux PF (1988) Influence of chlorpromazine on the transverse mobility of phospholipids in the human erythrocyte membrane: relation to shape changes. Biochim Biophys Acta 942:271-279

Sackmann E, Duwe HP, Engelhard H (1986) Membrane bending elasticity and its role for the shape fluctuations and the shape transformations of cells and vesicles. Faraday Discuss Chem Soc $81: 281-290$

Sheetz MP, Singer SJ (1974) Biological membranes as bilayer couples. A molecular mechanism of drug-erythrocyte interactions. Proc Natl Acad Sci USA 71:4457-4461

Svetina S, Zeks B (1989) Membrane bending energy and shape determination of phospholipid vesicles and red blood cells. Eur Biophys J 17:101-111

Van Meer G (1987) Plasma membrane cholesterol pools. TIBS $12: 375-376$

Zachowski A, Devaux PF (1989) Bilayer asymmetry and lipid transport across biomembranes. Comm Mol Cell Biophys 6:63-90

Zeman K, Engelhard H, Sackmann E (1990) Bending ondulations and elasticity of the erythrocyte membrane: effects of cell shape and membrane organisation. Eur Biophys J 18:203-219 